\title{
Inverse Stable Subordinators
}

\author{
M. M. Meerschaert ${ }^{1}$, P. Straka ${ }^{2}$ \\ ${ }^{1}$ Department of Statistics and Probability, Michigan State University, East Lansing, MI 48824 \\ ${ }^{2}$ School of Mathematics, University of Manchester, Manchester, M13 9PL, United Kingdom
}

\begin{abstract}
The inverse stable subordinator provides a probability model for time-fractional differential equations, and leads to explicit solution formulae. This paper reviews properties of the inverse stable subordinator, and applications to a variety of problems in mathematics and physics. Several different governing equations for the inverse stable subordinator have been proposed in the literature. This paper also shows how these equations can be reconciled.
\end{abstract}

Keywords and phrases: fractional derivative, subordinator, governing equation, hitting time

Mathematics Subject Classification: 60E07, 35R11, 26A33

\section{Introduction}

Fractional differential equations have become an important and useful tool in many areas of science and engineering $[15,17,34]$. This literature includes important applications to physics $[20,21]$, finance $[19,36]$, and hydrology $[4,5]$. The mathematical theory of fractional calculus began with a letter from Leibniz to L'Hôpital in 1695, as a mathematical curiosity, but recent applications have intensified interest in the mathematical community $[26,32,35,43]$.

An interesting connection with probability theory illuminates the physical meaning of the fractional derivatives. The famous paper of Einstein [8] connects the diffusion equation $\partial_{t} p=\partial_{x}^{2} p$, Brownian motion, and a simple random walk. The time-fractional diffusion equation $\partial_{t}^{\beta} p=\partial_{x}^{2} p$ governs the limit of a random walk with waiting times between jumps [23]: The $n$th particle jump $X_{n}$ is preceded by a waiting time $W_{n}$ with a power law probability distribution $P\left(W_{n}>t\right) \approx t^{-\beta}$ for some $0<\beta<1$. This random walk model converges to a Brownian motion $B(t)$, with the time index $t$ replaced by a random clock $E_{t}$. The resulting process $B\left(E_{t}\right)$ is sub-diffusive, spreading at a slower rate $t^{\beta / 2}$ than the usual rate $t^{1 / 2}$ for a traditional Brownian motion. Explicit solutions to the time-fractional diffusion equation can be obtained by a conditioning argument (see Eq. (2.7) below), using the probability density for the inverse stable subordinator $E_{t}$. The resulting solutions are useful to model diffusive phenomena in which particles rest for long periods between movements. This link between probability and differential equations has proven useful as the basis for particle tracking, a popular numerical method for solving certain partial differential equations $[16,45]$.

The purpose of this paper is to review the basic ideas behind the inverse stable subordinator $E_{t}$, as a probability model for time-fractional differential equations. The simplest such equation is the one that

*Corresponding author. E-mail: mcubed@unr.edu 
governs the process $E_{t}$ itself. A number of seemingly different governing equations have been reported in the literature. In this paper, we will review the various approaches, and show how they can be reconciled, by carefully investigating the different kinds of (ordinary and fractional) derivatives used in those equations.

\section{Time-fractional diffusion}

The random walk $S(n)=X_{1}+\cdots+X_{n}$ is a model for diffusive particle movements. The random variable $X_{n}$ represents the $n$th particle jump, and the sum $S(n)$ gives the particle location after $n$ jumps. The central limit theorem [26, Theorem 3.36] shows that the long-time limit of this random walk is a Brownian motion: $n^{-1 / 2} S([n t]) \Rightarrow B(t)$ in distribution, where $B(t)$ is normal with mean zero and variance $\sigma^{2} t$. This assumes that the jumps $X_{n}$ are independent and identically distributed with variance $\sigma^{2}$ and mean zero. The probability density function

$$
p(x, t)=\frac{1}{\sigma \sqrt{2 \pi t}} e^{-x^{2} /\left(2 \sigma^{2} t\right)}
$$

of $B(t)$ is the unique point source solution to the diffusion equation

$$
\partial_{t} p=D \partial_{x}^{2} p
$$

where $2 \sigma^{2}=D$. This can easily be checked using the Fourier transform $\hat{p}(k, t)=\int e^{-i k x} p(x, t) d x$ : Recall that $(i k) \hat{f}(k)$ is the Fourier transform of $\partial_{x} f(x)$, and take Fourier transforms in the diffusion equation (2.2) to get

$$
\partial_{t} \hat{p}(k, t)=D(i k)^{2} \hat{p}(k, t)=-D k^{2} \hat{p}(k, t) .
$$

Using the point source initial condition $p(x, 0)=\delta(x)$, and noting that $\hat{p}(k, 0) \equiv 1$, the unique solution to the ordinary differential equation $(2.3)$ is given by $\hat{p}(k, t)=\exp \left(-D t k^{2}\right)$. Inverting this Fourier transform [41, p. 524] leads back to (2.1).

In a continuous time random walk $[23,28,39]$, a random waiting time $W_{n}$ precedes the random particle jump $X_{n}$. Now $T_{n}=W_{1}+\cdots+W_{n}$ is the time of the $n$th particle jump. The number of jumps by time $t>0$ is $N_{t}=\max \left\{n \geq 0: T_{n} \leq t\right\}$, and the particle location at time $t>0$ is therefore $S\left(N_{t}\right)$. If the mean waiting time $\mu=\mathbb{E}\left[W_{n}\right]$ is finite, then the renewal theorem [7, Theorem 2.4.6] shows that $N_{t} / t \rightarrow \lambda=1 / \mu$ with probability one as $t \rightarrow \infty$, and the long-time limit of the particle location is another Brownian motion $B(\lambda t)$. However, for power law distributed waiting times, with $P\left(W_{n}>t\right) \sim C t^{-\beta}$ as $t \rightarrow \infty$ for some $0<\beta<1$, the mean diverges, and the extended central limit theorem [26, Theorem 3.37] shows that $n^{-1 / \beta} T_{[n u]} \Rightarrow D_{u}$, a stable subordinator whose probability density $g(x, u)$ has Laplace transform

$$
\tilde{g}(s, u)=\int_{0}^{\infty} e^{-s t} g(t, u) d t=\exp \left(-u C \Gamma(1-\beta) s^{\beta}\right) .
$$

For $C=1 / \Gamma(1-\beta)$, the Laplace transform simplifies to $\tilde{g}(s, u)=\exp \left(-u s^{\beta}\right)$. This is called the standard stable subordinator. The inverse process has an inverse limit [23, Theorem 3.2]: $n^{-\beta} N_{n t} \Rightarrow E_{t}$ where the inverse stable subordinator

$$
E_{t}=\inf \left\{u>0: D_{u}>t\right\}
$$

is also the first passage time of the process $D_{u}$ above the level $t>0$. An explicit formula for the moments of $E_{t}$ was given by Piryatinska, Saichev and Woyczynski [31]. Now it follows [23, Theorem 4.2] that

$$
n^{-\beta / 2} S\left(N_{t}\right)=\left(n^{-\beta}\right)^{-1 / 2} S\left(n^{\beta} n^{-\beta} N_{t}\right) \approx\left(n^{-\beta}\right)^{-1 / 2} S\left(n^{\beta} E_{t}\right) \Rightarrow B\left(E_{t}\right) .
$$

Figure 1 shows a typical particle trace (sample path) for this process, simulated using R [33] (see [26, Section 5.2] for more details). The graph in Figure 1 resembles a Brownian motion, with occasional long resting periods between particle movements. 


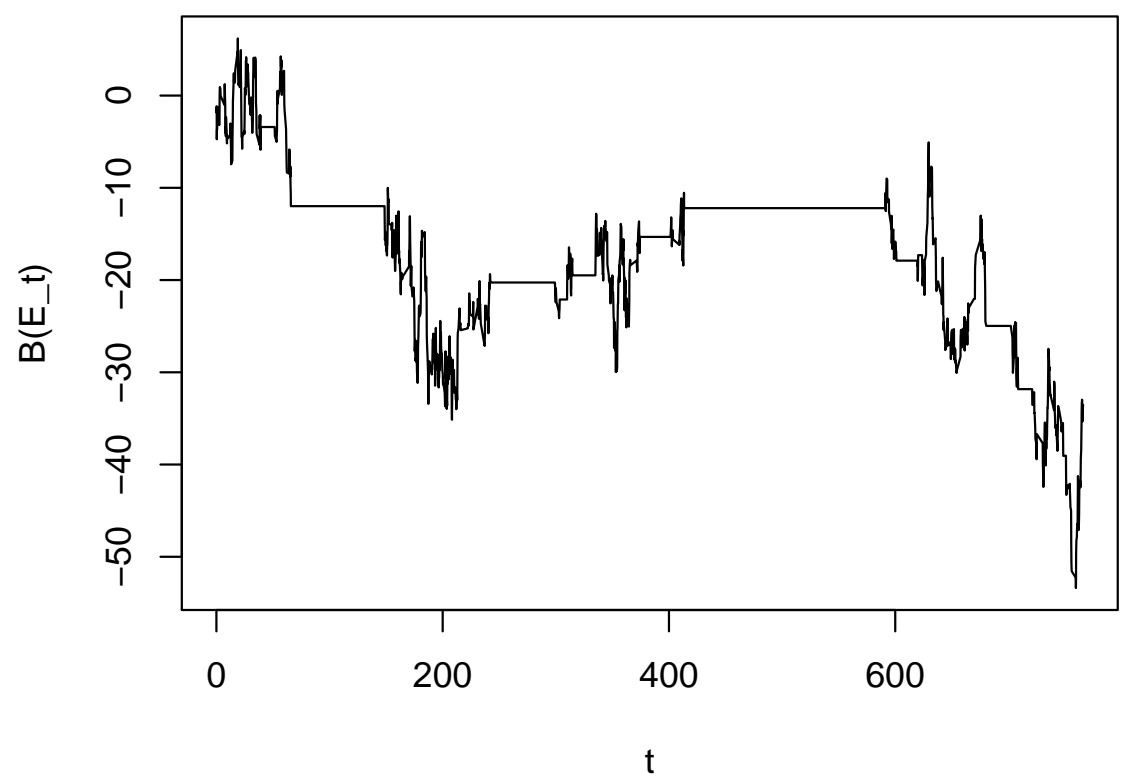

Figure 1. Typical sample path of the time-fractional diffusion process $B\left(E_{t}\right)$ from (2.6) with $D=0.5$ and $\beta=0.8$.

A simple conditioning argument shows that the stochastic limit $B\left(E_{t}\right)$ has density function

$$
q(x, t)=\int_{0}^{\infty} p(x, u) h(u, t) d u
$$

where $h(u, t)$ is the density of $E_{t}$. Since $t=D_{u}$ and $u=E_{t}$ are inverse processes, we have $P\left(E_{t} \leq u\right)=$ $P\left(D_{u} \geq t\right)$, and hence

$$
h(u, t)=\partial_{u} P\left(E_{t} \leq u\right)=\partial_{u}\left[1-P\left(D_{u}<t\right)\right]=-\partial_{u} \int_{0}^{t} g(y, u) d y
$$

Since $D_{u}$ has the same distribution as $u^{1 / \beta} D(1)$, we can write $g(t, u)=u^{-1 / \beta} g_{\beta}\left(t u^{-1 / \beta}\right)$, where $\tilde{g}_{\beta}(s)=$ $\exp \left(-s^{\beta}\right)$. Then a simple computation [23, Corollary 3.1] shows that

$$
h(u, t)=\frac{t}{\beta} u^{-1-1 / \beta} g_{\beta}\left(t u^{-1 / \beta}\right) .
$$

Figure 2 plots a typical density $h(x, t)$. Note that the density is zero for $x<0$ (since $E_{t}>0$ for $t>0$ ), with a discontinuity at $x=0$. Take Laplace transforms in (2.8), recalling that $s^{-1} \tilde{f}(s)$ in the Laplace transform of $\int_{0}^{t} f(y) d y$, to see that

$$
\tilde{h}(u, s)=-\partial_{u}\left[s^{-1} \exp \left(-u s^{\beta}\right)\right]=s^{\beta-1} \exp \left(-u s^{\beta}\right) .
$$




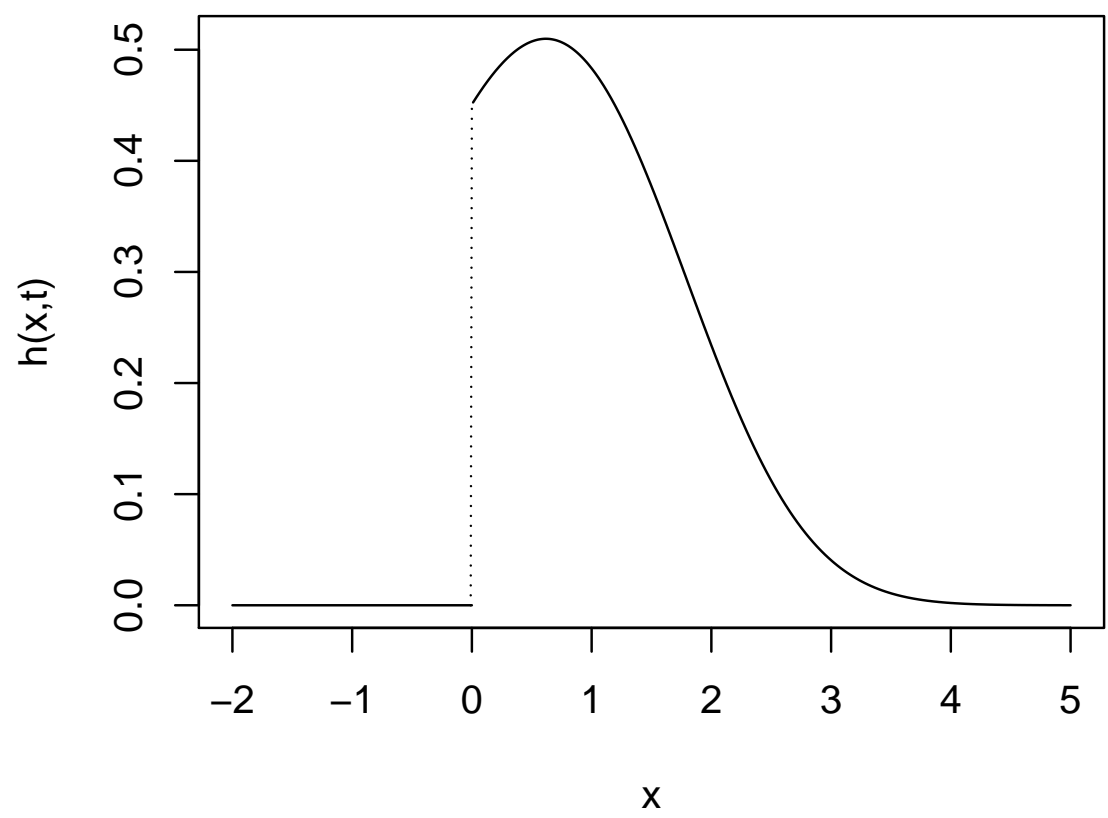

Figure 2. Inverse stable density $h(x, t)$ from $(2.9)$ with $\beta=0.6$ and $t=1$.

Now take Fourier transforms as well, to see that

$$
\begin{aligned}
\bar{q}(k, s) & =\int e^{-i k x} \tilde{q}(x, s) d x=\int_{0}^{\infty} \hat{p}(k, u) \tilde{h}(u, s) d u \\
& =\int_{0}^{\infty} e^{-u D k^{2}} s^{\beta-1} e^{-u s^{\beta}} d u=\frac{s^{\beta-1}}{s^{\beta}+D k^{2}} .
\end{aligned}
$$

Rewrite in the form $s^{\beta} \bar{q}=-D k^{2} \bar{q}+s^{\beta-1}$. Invert the Fourier transform to get

$$
s^{\beta} \tilde{q}(x, s)=D \partial_{x}^{2} \tilde{q}(x, s)+s^{\beta-1} \delta(x) .
$$

Use the definition of the gamma function to check that $f(t)=t^{-\beta} / \Gamma(1-\beta)$ has Laplace transform $\tilde{f}(s)=s^{\beta-1}$ for any $\beta>0$. Define the Riemann-Liouville fractional derivative $\mathbb{D}_{t}^{\beta} f(t)$ as the function with Laplace transform $s^{\beta} \tilde{f}(s)$. Now invert the Laplace transform to obtain the time-fractional diffusion equation $[12,40,44]$

$$
\mathbb{D}_{t}^{\beta} q(x, t)=D \partial_{x}^{2} q(x, t)+\frac{t^{-\beta}}{\Gamma(1-\beta)} \delta(x) .
$$

The conditioning formula (2.7) along with (2.1) and (2.9) gives an explicit solution in terms of the stable density. Figure 3 plots a typical solution curve. The sharp peak is the result of particles that get stuck at or near the source, due to long waiting times between movements. 


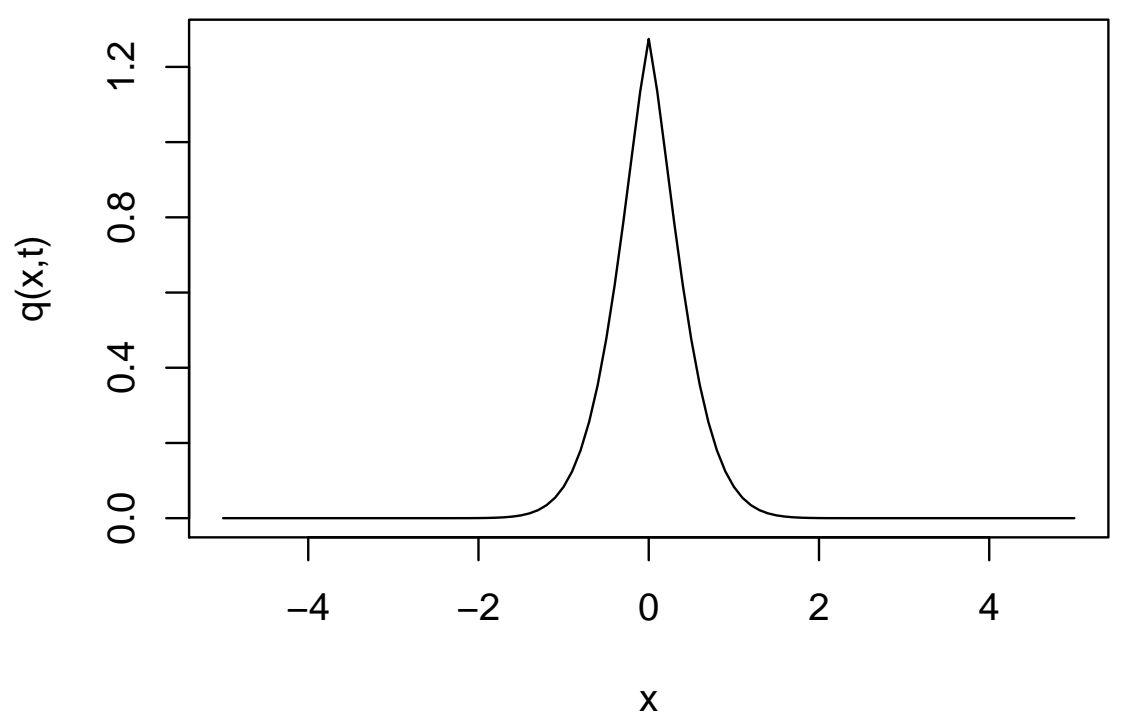

Figure 3. Solution (2.7) to time-fractional diffusion equation (2.11) at time $t=0.1$ with $\beta=0.8$ and $D=1.0$.

\section{Fractional derivatives}

In the previous section, we defined the Riemann-Liouville fractional derivative $\mathbb{D}_{t}^{\beta} f(t)$ as the function with Laplace transform $s^{\beta} \tilde{f}(s)$. The Caputo fractional derivative $\partial_{t}^{\beta} f(t)$ can be defined as the function with Laplace transform $s^{\beta} \tilde{f}(s)-s^{\beta-1} f(0+)$. When $\beta=1$, this is the traditional first derivative. Since $s^{\beta-1}$ is the Laplace transform of $t^{-\beta} / \Gamma(1-\beta)$, it follows that these two derivatives are related by

$$
\partial_{t}^{\beta} f(t)=\mathbb{D}_{t}^{\beta} f(t)-\frac{t^{-\beta}}{\Gamma(1-\beta)} f(0+) .
$$

A more general definition, without resorting to transforms, uses fractional integration. The RiemannLiouville fractional integral of order $\beta>0$ is defined by the formula

$$
\mathbb{I}^{\beta} f(t)=\frac{1}{\Gamma(\beta)} \int_{0}^{\infty} f(t-r) r^{\beta-1} d r
$$

Fractional integration satisfies the semigroup property $\mathbb{I}^{\beta} \mathbb{I}^{\alpha} f(t)=\mathbb{I}^{\beta+\alpha} f(t)$, and $\mathbb{I}^{1} f(t)=\int_{-\infty}^{t} f(u) d u$ is the traditional first integral. The Riemann-Liouville fractional derivative of order $\beta \in(0,1]$ is defined by

$$
\mathbb{D}_{t}^{\beta} f(t)=\partial_{t} \mathbb{I}_{t}^{1-\beta} f(t)=\frac{d}{d t} \frac{1}{\Gamma(1-\beta)} \int_{0}^{\infty} f(t-r) r^{-\beta} d r .
$$

If $f$ is a bounded function, then $\mathbb{I}_{t}^{1-\beta} f(0+)=0$, and it follows, using the formula for the Laplace transform of the first derivative, that the right-hand side of equation (3.3) has Laplace transform $s^{\beta} \tilde{f}(s)$. More generally, the Laplace transform of $\mathbb{D}_{t}^{\beta} f(t)$ is $s^{\beta} \tilde{f}(s)-s^{\beta-1} \mathbb{I}_{t}^{1-\beta} f(0+)$. The Caputo fractional derivative 
of order $\beta \in(0,1]$ is defined by

$$
\partial_{t}^{\beta} f(t)=\mathbb{I}_{t}^{1-\beta} \partial_{t} f(t)=\frac{1}{\Gamma(1-\beta)} \int_{0}^{\infty} f^{\prime}(t-r) r^{-\beta} d r .
$$

Some authors (e.g., see [12]) use (3.1) as the definition of the Caputo derivative, since it exists for a broader class of functions $f$. Since $f^{\prime}$ has Laplace transform $s \tilde{f}(s)-f(0+)$, it follows from the convolution formula for Laplace transforms that the right-hand side of (3.4) has Laplace transform $s^{\beta-1}[s \tilde{f}(s)-f(0+)]=$ $s^{\beta} \tilde{f}(s)-s^{\beta-1} f(0+)$. If $f(0+)=0$, then the two forms (3.3) and (3.4) are equivalent.

\subsection{Weak derivatives}

In the theory of differential equations, it is common to consider distributional solutions, where derivative operators are interpreted in a weak sense. Let $C_{c}^{\infty}(\mathbb{R})$ denote the topological vector space of infinitely differentiable real-valued functions on $\mathbb{R}$ with compact support (test functions). A distribution is a continuous linear functional $f: C_{c}^{\infty}(\mathbb{R}) \rightarrow \mathbb{R}$. Here continuity means [42] that $f\left(\phi_{n}\right) \rightarrow 0$ for any sequence $\left(\phi_{n}\right)$ in $C_{c}^{\infty}$ such that $\partial^{k} \phi_{n} \rightarrow 0$ as $n \rightarrow \infty$ for all $k \geq 0$, and every $\phi_{n}$ is supported on the same compact set $K$. Any locally integrable function $f$ on $\mathbb{R}$ defines a distribution $f(\phi)=\int f(x) \phi(x) d x$. Any $\sigma$ finite Borel measure $\mu$ defines a distribution $\int \phi(x) \mu(d x)$. The Dirac delta function $\int \delta(x) \phi(x) d x=\phi(0)$ corresponds to a Borel measure that assigns unit mass to the point $x=0$. Any distribution $f$ has a derivative $\mathfrak{D}_{x} f$, another distribution defined via integration by parts: $\mathfrak{D}_{x} f(\phi)=-\int f(x) \phi^{\prime}(x) d x$. If $f(x)$ is a differentiable function, then the distributional (weak) derivative is the distribution associated with the ordinary derivative.

Suppose that: $f(x)$ is differentiable at every point $x>0 ; f(x)=0$ for $x<0$; and the right limit $f(0+)$ exists. Then $\mathfrak{D}_{x} f(x)$ is defined by

$$
\mathfrak{D}_{x} f(x)=f^{\prime}(x)+f(0+) \delta(x) .
$$

Equation (3.5) can be used to explain the difference between the standard formulae for the Laplace and Fourier transforms of the first derivative $f^{\prime}$. The Laplace transform of $f^{\prime}$ is given by

$$
\begin{aligned}
\int_{0}^{\infty} e^{-s t} f^{\prime}(t) d t & =\left.e^{-s t} f(t)\right|_{t=0+} ^{\infty}+\int_{0}^{\infty} s e^{-s t} f(t) d t \\
& =-e^{-0} f(0+)+s \tilde{f}(s)=s \tilde{f}(s)-f(0+)
\end{aligned}
$$

assuming that $e^{-s t} f(t) \rightarrow 0$ as $t \rightarrow \infty$. If we interpret the Fourier transform as a traditional integral, ignoring the jump at $x=0$, then since $f^{\prime}=0$ for $x<0$, we get the analogous result:

$$
\begin{aligned}
\int_{-\infty}^{\infty} e^{-i k x} f^{\prime}(x) d x & =\int_{0}^{\infty} e^{-i k x} f^{\prime}(x) d x \\
& =\left.e^{-i k x} f(x)\right|_{x=0+} ^{\infty}+\int_{0}^{\infty}(i k) e^{-i k x} f(x) d x \\
& =-e^{-0} f(0+)+(i k) \hat{f}(k)=(i k) \hat{f}(k)-f(0+)
\end{aligned}
$$

assuming that $f(x) \rightarrow 0$ as $x \rightarrow \infty$. Indeed, this formula can be interpreted as the Laplace transform of $f^{\prime}$ evaluated at the complex argument $s=i k$. In many applications [1,46] it is common to consider Laplace transforms with complex arguments. However, the usual interpretation of the derivative in this situation (when the Fourier transform is applied) is in terms of distributions. In that sense, we see that

$$
\begin{aligned}
\int_{-\infty}^{\infty} e^{-i k x} \mathfrak{D}_{x} f(x) d x & =\int_{-\infty}^{\infty} e^{-i k x} f(0+) \delta(x) d x+\int_{0}^{\infty} e^{-i k x} f^{\prime}(x) d x \\
& =e^{-0} f(0+)+(i k) \hat{f}(k)-f(0+)=(i k) \hat{f}(k) .
\end{aligned}
$$


In the theory of fractional differential equations, it is also useful to interpret fractional derivatives as distributions. First, we define the convolution $f * g$ of two distributions $f$ and $g$. For our purposes, the case where $f$ and $g$ are locally integrable functions will suffice. Then $f * g$ is given via [37]

$$
f * g(\phi)=\iint f(x) g(y) \phi(x+y) d x d y
$$

and we note that this definition is valid whenever $f$ has compact support or when the support of both $f$ and $g$ is bounded below, e.g., when $f(x)=g(x)=0$ for $x<0$. The distribution $f * g$ can then be associated with the (locally integrable) function $x \mapsto \int f(x-y) g(y) d y$. Next, we note that, for a distribution $f$, the fractional integral (3.2) can be interpreted as the distribution $f * k_{\beta}$, where $k_{\beta}(x)=I(x>0) x^{\beta-1} / \Gamma(\beta)$. Since convolution and weak derivative commute, that is $\mathfrak{D}(f * g)=(\mathfrak{D} f) * g$, replacing the derivative $\partial_{t}$ in (3.3) and (3.4) by the weak derivative $\mathfrak{D}_{t}$ yields the same distribution. Hence within the framework of distributional calculus, we do not need to distinguish between the Riemann-Liouville and Caputo forms, and we refer to $\mathfrak{D}^{\beta} f:=\mathfrak{D}\left[k_{1-\beta} * f\right]=k_{1-\beta} * \mathfrak{D} f$ as the weak fractional derivative of order $\beta$. This can also be seen via Fourier transforms: Since $k_{1-\beta}(x)$ has Fourier transform $(i k)^{\beta-1}$ in the sense of generalized functions [44], the convolution property of the Fourier transform implies that convolution with $k_{1-\beta}$ corresponds to a multiplication with $(i k)^{\beta-1}$ in Fourier space. As mentioned above, a weak derivative corresponds to a multiplication with $(i k)$ in Fourier space. The two different orderings of the two operations hence correspond to the same multiplication by $(i k)(i k)^{\beta-1}=(i k)^{\beta-1}(i k)=(i k)^{\beta}$ in Fourier space, and so $\mathfrak{D}_{x}^{\beta} f(x)$ has Fourier transform $(i k)^{\beta} \hat{f}(k)$. In the recent book [26], $\mathfrak{D}_{x}^{\beta} f(x)$ is called the generator form of the fractional derivative.

In this paper, we apply weak fractional derivatives to functions $f(x)$ that are differentiable at every point $x>0$, with $f(x)=0$ for $x<0$, and $f(0+)$ exists. Then $\mathbb{I}_{x}^{1-\beta} f(x)$ is differentiable at every point, except possibly $x=0$, and vanishes on $x<0$. Now we can use (3.5) to write the weak fractional derivative in the form

$$
\mathfrak{D}_{x}^{\beta} f(x)=\mathbb{D}_{x}^{\beta} f(x)+\delta(x) \mathbb{I}_{x}^{1-\beta} f(0+) .
$$

If $f(x)$ is bounded, then $\mathbb{I}_{x}^{1-\beta} f(x) \rightarrow 0$ as $x \rightarrow 0+$, and the Riemann-Liouville fractional derivative equals the weak fractional derivative.

\section{The inverse stable density}

The explicit solution (2.7) to the time-fractional diffusion equation (2.11) involves the probability density $h(x, t)$ of the inverse stable subordinator (2.5). Equation (2.9) expresses $h(x, t)$ for all $t>0$ and $x>0$ in terms of the density $g_{\beta}$ of a standard stable subordinator. The function $g_{\beta}(u)$ is infinitely differentiable on the entire real line, with $g_{\beta}(u)=0$ for all $u \leq 0$, see Zolotarev [46]. For $x<0$ or $t<0$, we will define $h(x, t)=0$. At the boundary of the first quadrant, we will define $h(x, t)$ to be right-continuous: Equation $(2.2)$ in [10] (see also [43]) shows that

$$
g_{\beta}(u) \sim K(\beta / u)^{(1-\beta / 2) /(1-\beta)} \exp \left\{-|1-\beta|(u / \beta)^{\beta /(\beta-1)}\right\} \quad \text { as } u \rightarrow 0+
$$

where $K=1 / \sqrt{2 \pi \beta(1-\beta)}>0$. It follows that $h(x, t) \rightarrow 0$ as $t \rightarrow 0+$, so that $h(x, t)$ is a continuous function of $t \in \mathbb{R}$ for all $x>0$.

Furthermore, we have

$$
g_{\beta}(u) \sim \frac{\beta}{\Gamma(1-\beta)} u^{-\beta-1} \text { as } u \rightarrow \infty,
$$

as noted for example in (2.3) of [10]. Then it follows from (2.9) and (4.2) that

$$
h(x, t) \sim \frac{t}{\beta} x^{-1-1 / \beta} \frac{\beta}{\Gamma(1-\beta)}\left(t x^{-1 / \beta}\right)^{-\beta-1} \rightarrow \frac{t^{-\beta}}{\Gamma(1-\beta)} \quad \text { as } x \rightarrow 0+
$$

for all $t>0$. This jump in the function $x \mapsto h(x, t)$ was illustrated in Figure 2. 
The governing equation for $h(x, t)$ involves a fractional derivative in the $t$ variable, and a derivative of order one in the $x$ variable. Suppose that $x>0$. Since $h(x, t)$ is a bounded function of $t \in \mathbb{R}$, it follows from [26, Section 2.3] that the Riemann-Liouville fractional derivative $\mathbb{D}_{t}^{\beta} h(x, t)$ exists, and its Laplace transform equals $s^{2 \beta-1} \exp \left(-x s^{\beta}\right)$. Since $g_{\beta}(u)$ is a smooth function of $u \in \mathbb{R}$ that vanishes on $u \leq 0$, it follows from $(2.9)$ that $h(x, t)=0$ for $x>0$ and $t \leq 0$. Then $\mathbb{D}_{t}^{\beta} h(x, t)=0$ when $x>0$ and $t<0$. Since $h(x, 0+)=0$ for $x>0$, it follows from (3.1) that the Caputo fractional derivative $\partial_{t}^{\beta} h(x, t)$ equals the Riemann-Liouville form when $x>0$. Since $h(x, t)$ is a bounded function of $t \in \mathbb{R}$, equation (3.6) implies that the weak fractional derivative $\mathfrak{D}_{t}^{\beta} h(x, t)$ equals the Riemann-Liouville fractional derivative $\mathbb{D}_{t}^{\beta} h(x, t)$ when $x \neq 0$.

If $t>0$, then $h(x, t)$ is a differentiable function of $x \in \mathbb{R}$ except for a jump at $x=0$. Using (4.3), it follows from (3.5) that

$$
\mathfrak{D}_{x} h(x, t)=\partial_{x} h(x, t)+\frac{t^{-\beta}}{\Gamma(1-\beta)} \delta(x)
$$

for all $t>0$. If $t<0$, then we have defined $h(x, t)=0$ for all $x \in \mathbb{R}$, so both the weak and traditional derivatives are identically zero.

\section{Governing equations}

In this section, we review several seemingly different governing equations for the inverse stable density $h(x, t)$ that have appeared in the mathematics and physics literature. By carefully considering the different kinds of (ordinary and fractional, weak and traditional) derivatives used, we will show how all these equations can be reconciled. Along the way, we will review several important applications of the inverse stable subordinator to the general theory of time-fractional diffusion.

\subsection{Semigroup approach}

The inverse stable density (2.9) can be used to solve fractional Cauchy problems. A family of linear operators $\left\{T_{t}: t \geq 0\right\}$ on a Banach space $X$ of functions is called a $C_{0}$ semigroup if $T_{0} f(x)=f(x)$, $T_{t} T_{s} f(x)=T_{t+s} f(x),\left\|T_{t} f(x)-f(x)\right\| \rightarrow 0$ in the Banach space norm as $t \rightarrow 0$, and for each $t \geq 0$ there exists a constant $M_{t}>0$ such that $\left\|T_{t} f(x)\right\| \leq M_{t}\|f(x)\|$ for every function $f(x)$ in the Banach space $[1,30]$. Every $C_{0}$ semigroup has a generator

$$
L f(x)=\lim _{t \rightarrow 0} \frac{T_{t} f(x)-f(x)}{t}
$$

defined for $f \in \operatorname{Dom}(L)$, a dense subset of $X$, and $p(x, t)=T_{t} f(x)$ solves the Cauchy problem

$$
\partial_{t} p(x, t)=L p(x, t) ; \quad p(x, 0)=f(x)
$$

for any $f \in \operatorname{Dom}(L)$.

Time-fractional Cauchy problems were first considered in $[12,13,29,40]$. For any $C_{0}$ semigroup, Theorem 3.1 in [2] shows that the fractional Cauchy problem

$$
\mathbb{D}_{t}^{\beta} q(x, t)=L q(x, t)+\frac{t^{-\beta}}{\Gamma(1-\beta)} f(x)
$$

has solution (2.7) for every $f \in \operatorname{Dom}(L)$, where $p(x, u)$ is the corresponding solution to the Cauchy problem (5.1), and $h(u, t)$ is given by (2.9). Since $h(u, t)$ is the probability density of $E_{t}$, we can also write $q(x, t)=\mathbb{E}\left[p\left(x, E_{t}\right)\right]$, and since $E_{0}=0$, this shows that $q(x, 0)=p(x, 0)=f(x)$, i.e., the fractional Cauchy problem (5.2) has the same initial data as the Cauchy problem (5.1). 
The governing equation for $h(x, t)$ follows as a special case of (5.2). First note that the shift semigroup $T_{t} f(x)=f(x-t)$ has generator $L f(x)=-\mathfrak{D}_{x} f(x)$ using the weak derivative: For any test function $\phi(x)$, write

$$
\begin{aligned}
L f(\phi) & =\int L f(x) \phi(x) d x \\
& =\int\left[\lim _{t \downarrow 0} \frac{f(x-t)-f(x)}{t}\right] \phi(x) d x \\
& =\lim _{t \downarrow 0} \frac{1}{t}\left[\int f(x) \phi(x+t) d x-\int f(x) \phi(x) d x\right] \\
& =\int f(x) \phi^{\prime}(x) d x=f\left(\phi^{\prime}\right)=-\mathfrak{D}_{x} f(\phi) .
\end{aligned}
$$

For example, we can work on the Banach space of bounded continuous functions that vanish at infinity, with the supremum norm. Then $p(x, t)=T_{t} f(x)=f(x-t)$ solves the Cauchy problem $\partial_{t} p(x, t)=$ $-\mathfrak{D}_{x} p(x, t)$ with initial condition $p(x, 0)=f(x)$. Apply [2, Theorem 3.1] to see that $(2.7)$ with $p(x, t)=$ $f(x-t)$ solves the fractional Cauchy problem (5.2) with $L=-\mathfrak{D}_{x}$. Next we will show that $h(x, t)$ solves the same fractional Cauchy problem, with a point source initial condition, in the distributional sense.

Assume, without loss of generality, that $f$ has compact support. Then it is easy to check that $q(x, t)$ from (2.7) with $p(x, t)=f(x-t)$ satisfies $q(x, t)=h(x, t) *[f(x) \delta(t)]$ in the sense of distributions on $\mathbb{R}^{2}$ (the convolution is taken in both variables). Since convolution and partial derivatives commute in the framework of distributional calculus, we find using (5.2)

$$
\left[\left(\mathbb{D}_{t}^{\beta}+\mathfrak{D}_{x}\right) h(x, t)\right] *[f(x) \delta(t)]=\left(\mathbb{D}_{t}^{\beta}+\mathfrak{D}_{x}\right) q(x, t)=f(x) \frac{t^{-\beta}}{\Gamma(1-\beta)} .
$$

Applying left and right-hand side to $\mathbb{R}^{2}$-test functions of the form $\phi(x) \psi(t)$, it follows that $m(x, t):=$ $\left(\mathbb{D}_{t}^{\beta}+\mathfrak{D}_{x}\right) h(x, t)$ must satisfy

$$
\int m(x-y, t) f(y) d y=f(x) \frac{t^{-\beta}}{\Gamma(1-\beta)}
$$

for almost all $(x, t) \in \mathbb{R}^{2}$. But then for almost every $t \in \mathbb{R}$ necessarily $m(x, t)=\delta(x) \frac{t^{-\beta}}{\Gamma(1-\beta)}$, as $f$ was chosen arbitrarily from the dense domain of $L$. It follows that $h(x, t)$ solves the fractional Cauchy problem

$$
\mathbb{D}_{t}^{\beta} h(x, t)=-\mathfrak{D}_{x} h(x, t)+\frac{t^{-\beta}}{\Gamma(1-\beta)} \delta(x)
$$

in the sense of distributions. Since $q(x, 0)=\int_{0}^{\infty} f(x-u) h(u, 0) d u=f(x)$, the initial data is $h(x, 0)=$ $\delta(x)$.

\subsection{CTRW approach}

The inverse stable subordinator (2.5) emerges as the time change in a CTRW limit. A general triangular array scheme outlined in [24] leads to a CTRW limit process $A\left(E_{t}\right)$ with governing equation

$$
\mathbb{D}_{t}^{\beta} q(x, t)=-\psi\left(-i \mathfrak{D}_{x}\right) q(x, t)+\frac{t^{-\beta}}{\Gamma(1-\beta)} \delta(x) .
$$

The random walk of particle jumps converges in the limit to a Lévy process $A(u)$ with density $p(x, u)$, and Fourier transform $\hat{p}(k, u)=\exp (-u \psi(k))$. The pseudo-differential operator term $\psi\left(-i \mathfrak{D}_{x}\right) q(x, t)$ is defined as the function with Fourier transform $\psi(k) \hat{q}(k, t)$ (e.g., see Jacob [11]). For a CTRW with 
mean zero, finite variance jumps, the limit process $A(t)$ is a Brownian motion, $\psi(k)=D k^{2}$, the pseudodifferential operator $\psi\left(-i \mathfrak{D}_{x}\right)=D\left(-i \mathfrak{D}_{x}\right)^{2}=-D \mathfrak{D}_{x}^{2}$, and (5.6) reduces to a weak form of the timefractional diffusion equation (2.11).

The simplest CTRW uses a deterministic particle jump $X_{n}=1$. Then $x=A(u)=u$ is a ballistic motion, with "density" $p(x, u)=\delta(x-u)$, and the CTRW limit $A\left(E_{t}\right)$ equals the inverse stable subordinator $E_{t}$. Since $\hat{p}(k, u)=e^{-i k u}$, the Fourier symbol is $\psi(k)=i k$, and the pseudo-differential operator $\psi\left(-i \mathfrak{D}_{x}\right)=\mathfrak{D}_{x}$ is the (weak) first derivative. Since $q(x, t)=h(x, t)$, it follows from (5.6) that the inverse stable subordinator has governing equation (5.5). Since $h(x, 0)=\delta(x)$, the relation (3.1) between Caputo and Riemann-Liouville fractional derivatives can be used [24, Remark 4.8] to rewrite the governing equation (5.5) in a more compact form

$$
\partial_{t}^{\beta} h(x, t)=-\mathfrak{D}_{x} h(x, t) .
$$

\subsection{Hamiltonian chaos}

Saichev and Zaslavsky [38] derive the equation

$$
\frac{\partial^{\beta}}{\partial t^{\beta}} q(x, t)=L q(x, t)+\frac{t^{-\beta}}{\Gamma(1-\beta)} q(x, 0)
$$

as a model for Hamiltonian chaos, where $0<\beta<1$, and $L$ is the generator of the semigroup associated with the particle movement process. Taking $L f(x)=-\mathfrak{D}_{x} f(x)$ for ballistic motion, this reduces to an alternative governing equation

$$
\frac{\partial^{\beta}}{\partial t^{\beta}} h(x, t)=-\mathfrak{D}_{x} h(x, t)+\frac{t^{-\beta}}{\Gamma(1-\beta)} h(x, 0)
$$

for the inverse stable subordinator, as noted in [38, Eq. (B.14)]. The paper [38] also develops the explicit solution (2.7) and the density formula (2.9), but does not identify the underlying stochastic process $E_{t}$. The fractional derivative $\partial^{\beta} / \partial t^{\beta}$ in (5.9) is defined in [38, Eq. (A.10)] as a convolution of generalized functions

$$
\frac{\partial^{\beta}}{\partial t^{\beta}} f(t)=f * k_{-\beta}(t)=\int_{0}^{\infty} f(t-u) \frac{u^{-\beta-1}}{\Gamma(-\beta)} d u .
$$

The authors note that this integral does not exist in the traditional sense, since the kernel $k_{-\beta}(t)=I(t>$ $0) t^{-\beta-1} / \Gamma(-\beta)$ is not integrable at $t=0+$, and they offer an alternative form

$$
\frac{\partial^{\beta}}{\partial t^{\beta}} f(t)=\frac{1}{\Gamma(1-\beta)} \int \frac{d f(u)}{d u}(t-u)^{-\beta} d u .
$$

This appears to be a Caputo derivative, in which case (5.9) would contradict (5.7). However, examples in [38, Appendix A] make it clear that the authors intend $d f / d u$ to represent the weak derivative. Then $\partial^{\beta} / \partial t^{\beta}$ equals the weak fractional derivative:

$$
\frac{\partial^{\beta}}{\partial t^{\beta}} f(t)=\mathbb{I}_{t}^{1-\beta} \mathfrak{D}_{t} f(t)=\frac{1}{\Gamma(1-\beta)} \int \mathfrak{D}_{u} f(u)(t-u)^{-\beta} d u .
$$

Since the weak fractional and Riemann-Liouville fractional derivatives are equal for $x \neq 0$ (see Section 4 ), the left-hand side of (5.9) reduces to the traditional Riemann-Liouville fractional derivative $\mathbb{D}_{t}^{\beta} h(x, t)$ for all $x \neq 0$, and hence the governing equation (5.9) agrees with the form (5.5).

\subsection{Generalized CTRW}

Kolokoltsov [14] develops governing equations for the long-time limit of a CTRW-like process, where the jumps distribution depends on the current state $x$ of the random walk, so that $A(t)$ is a time-homogeneous 
Markov process. Assuming that the motion process $A(t)$ has a transition density $p(x, t ; y)$ conditional on $y=A(0)$, the generalized CTRW limit $A\left(E_{t}\right)$ has a transition density

$$
q(x, t ; y)=\int_{0}^{\infty} p(x, u ; y) h(u, t) d u .
$$

If the Markov process $A(t)$ is homogeneous in $y$, this reduces to the formula (2.7). The transition density solves a governing equation

$$
\mathcal{A}_{t}^{*} p(x, t ; y)=-L_{x}^{*} p(x, t ; y)+\mathcal{A}_{t}^{*} H(t) \delta(x-y),
$$

where $L_{x}^{*}$ is the forward semigroup generator for the Markov process $A(t), \mathcal{A}_{t}^{*}$ is the forward generator for the standard stable subordinator $D_{t}$, and $H(t)=I(t>0)$ is the Heaviside function. In some cases (e.g, the Ornstein-Uhlenbeck process) the steady-state transition density $p(x, u ; y)$ can be computed in closed form, and then (5.10) along with (2.9) gives an explicit solution to equation (5.11).

The forward generator [14, Proposition 4.1] of the standard stable subordinator is

$$
\mathcal{A}^{*} f(t)=\frac{1}{\Gamma(1-\beta)} \int_{0}^{\infty}[f(t-u)-f(t)] \beta u^{-\beta-1} d u
$$

Then it follows from [26, Example 3.24] that $\mathcal{A}^{*} f(t)=-\mathfrak{D}_{t}^{\beta} f(t)$, using the weak fractional derivative. From (5.12), it is easy to compute the boundary term

$$
\mathcal{A}_{t}^{*} H(t)=-\frac{t^{-\beta}}{\Gamma(1-\beta)} .
$$

Suppose for example that $A(t)$ is a Brownian motion with forward generator $D \partial_{x}^{2}$, as in Section 2. Then the generalized CTRW governing equation (5.11) reduces to the time-fractional diffusion equation (2.11). Kolokoltsov also derives a governing equation

$$
\mathcal{A}_{t}^{*} h(x, t)=\mathfrak{D}_{x} h(x, t)+\delta(x) \mathcal{A}_{t}^{*} H(t)
$$

for the hitting time density. Substituting $\mathcal{A}^{*}=-\mathfrak{D}_{t}^{\beta}$ and (5.13), this reduces immediately to (5.5).

\subsection{Stochastic differential equations}

Hahn, Kobayashi, and Umarov [10] develop stochastic differential equations

$$
X_{t}=X_{0}+\int_{0}^{t} b\left(X_{s}\right) d E_{s}+\int_{0}^{t} a\left(X_{s}\right) d B\left(E_{s}\right)
$$

driven by a time-changed Brownian motion $B\left(E_{t}\right)$. They also derive the governing (forward) equation

$$
\partial_{t}^{\beta} q(x, t)=L q(x, t) ; \quad v(x, 0)=\delta(x),
$$

for the transition density, using the Caputo fractional derivative in time, and the generator (pseudodifferential operator)

$$
L f=\sum_{i, j=1}^{d} a_{i j}(x) \frac{\partial^{2} f}{\partial x_{i} \partial x_{j}}+\sum_{i=1}^{d} b_{i}(x) \frac{\partial f}{\partial x_{i}} .
$$

Using the relation (3.1), this can be rewritten in the form

$$
\mathbb{D}_{t}^{\beta} q(x, t)=L q(x, t)+\frac{t^{-\beta}}{\Gamma(1-\beta)} \delta(x),
$$


which agrees with (5.6). The governing equation for the hitting time density $h(x, t)$ is given $[10$, Lemma 2.2 ] by

$$
\partial_{t}^{\beta} h(x, t)=-\partial_{x} q(x, t)-\frac{t^{-\beta}}{\Gamma(1-\beta)} \delta(x),
$$

which at first glance seems to contradict (5.5), due to the negative sign in front of the $\delta(x)$ term. To reconcile with (5.5), first apply (3.5) to rewrite (5.16) in the form

$$
\partial_{t}^{\beta} h(x, t)=-\mathfrak{D}_{x} q(x, t) .
$$

Next, use the fact that $h(0+, t)=t^{-\beta} / \Gamma(1-\beta)$ along with (3.1) to arrive at (5.5). Equation (5.16) differs from (5.5) in two ways: It uses a Caputo derivative in $t$, rather than the Riemann-Liouville derivative; and it uses a strong derivative in $x$, rather than the weak derivative.

\subsection{Fractional Fokker-Planck equation}

Barkai, Metzler and Klafter [3] derive the fractional Fokker-Planck equation

$$
\partial_{t} q(x, t)=\mathbb{D}_{t}^{1-\beta} L q(x, t)+\delta(x) \delta(t)
$$

where $0<\beta<1$, and $L=D \partial_{x}^{2}-c \partial_{x} F(x)$ is the Fokker-Planck operator for motion and dispersion in a spatially varying external field. The fractional derivative accounts for particle sticking and trapping. The model is derived from a generalized CTRW model in [27]. If $A(t)$ is the Markov process with forward generator $L$, then the fractional Fokker-Planck equation (5.17) governs a time-changed Markov process $A\left(E_{t}\right)$. In the special case $L=-\mathfrak{D}_{x}$, the fractional Fokker-Planck equation models ballistic motion at unit speed, interrupted by random particle waiting times $W_{n}$ that follow a power law distribution $P\left(W_{n}>t\right) \approx t^{-\beta}$ for $t>0$ sufficiently large. Then the equation

$$
\partial_{t} h(x, t)=-\mathbb{D}_{t}^{1-\beta} \mathfrak{D}_{x} h(x, t)+\delta(x) \delta(t)
$$

should govern the inverse stable subordinator $h(x, t)$.

Now we reconcile equation (5.18) with (5.5): It follows from definition (3.3) that the Riemann-Liouville fractional derivative $\mathbb{D}_{t}^{1-\beta} f(t)=\partial_{t} \mathbb{I}_{t}^{\beta} f(t)$, the first derivative of a fractional integral. Integrate both sides of equation (5.18) from 0 to $t$, and recall that $h(x, 0+)=0$ (see Section 3.1), to obtain

$$
h(x, t)=-\mathbb{I}_{t}^{\beta} \mathfrak{D}_{x} h(x, t)+\delta(x) H(t),
$$

where $H(t)=I(t>0)$ is the Heaviside function. Next, apply the fractional integral (3.2) of order $1-\beta$ on both sides, compute $I_{t}^{1-\beta} H(t)=t^{1-\beta} / \Gamma(2-\beta)$, and use the semigroup property of the fractional integral (see Section 2) to get

$$
\mathbb{I}_{t}^{1-\beta} h(x, t)=-\mathbb{I}_{t}^{1} \mathfrak{D}_{x} h(x, t)+\delta(x) \frac{t^{1-\beta}}{\Gamma(2-\beta)} .
$$

Now take the first derivative $\partial_{t}$ on both sides, and use (3.3) again, to arrive at (5.5).

\subsection{Laplace and Fourier transforms}

Recall from (2.10) that the probability density $h(x, t)$ of the inverse stable subordinator $E_{t}$ has Laplace transform $\tilde{h}(x, s)=s^{\beta-1} \exp \left(-x s^{\beta}\right)$. Taking Laplace transforms in the $x$ variable as well, we arrive at the double Laplace transform

$$
\begin{aligned}
\bar{h}(\lambda, s) & =\int_{0}^{\infty} e^{-\lambda x} \tilde{h}(x, s) d x \\
& =\int_{0}^{\infty} e^{-\lambda x} s^{\beta-1} e^{-x s^{\beta}} d x=\frac{s^{\beta-1}}{s^{\beta}+\lambda} .
\end{aligned}
$$


Rewrite in the form

$$
s^{\beta} \bar{h}(k, s)=-\lambda \bar{h}(k, s)+s^{\beta-1}
$$

and note that $\tilde{h}(0+, s)=s^{\beta-1}$, so that $\lambda \bar{h}(k, s)-s^{\beta-1}$ is the Laplace transform $(x \mapsto \lambda)$ of the function $\partial_{x} \tilde{h}(x, s)$. Invert this Laplace transform to arrive at

$$
s^{\beta} \tilde{h}(x, s)=-\partial_{x} \tilde{h}(x, s) .
$$

Now use the fact that $s^{\beta} \tilde{f}(s)$ is the Laplace transform of the Riemann-Liouville fractional derivative $\mathbb{D}_{t}^{\beta} f(t)$ to invert the remaining transform:

$$
\mathbb{D}_{t}^{\beta} h(x, t)=-\partial_{x} h(x, t) .
$$

Next, use the relation (3.1) along with $h(x, 0)=\delta(x)$ to arrive at

$$
\partial_{t}^{\beta} h(x, t)+\frac{t^{-\beta}}{\Gamma(1-\beta)} \delta(x)=-\partial_{x} h(x, t) .
$$

Finally, move the delta function term to the opposite side, to obtain equation (5.16) from Hahn, Kobayashi, and Umarov [10].

Now follow the same steps, but use the Fourier transform instead of the Laplace transform in the $x$ variable: Write the Fourier-Laplace transform

$$
\check{h}(k, s)=\int_{0}^{\infty} e^{-i k x} s^{\beta-1} e^{-x s^{\beta}} d x=\frac{s^{\beta-1}}{s^{\beta}+i k} .
$$

Rewrite in the form

$$
s^{\beta} \breve{h}(k, s)-s^{\beta-1}=-i k \breve{h}(k, s)
$$

and invert the Fourier transform to get

$$
s^{\beta} \tilde{h}(x, s)-s^{\beta-1} \delta(x)=-\mathfrak{D}_{x} \tilde{h}(x, s) .
$$

Now invert the Laplace transform to arrive at (5.7), using the fact that $s^{\beta} \tilde{f}(s)-s^{\beta-1} f(0+)$ is the Laplace transform of the Caputo fractional derivative $\partial_{t}^{\beta} f(t)$, or the equivalent form (5.5), using the Laplace formula for the Riemann-Liouville derivative, along with the fact that $s^{\beta-1}$ is the Laplace transform of $t^{-\beta} / \Gamma(1-\beta)$.

The governing equations that result from the Laplace-Laplace or Fourier-Laplace transforms seem incompatible, until one remembers that the Fourier transform pair $(i k) \hat{f}(x) \leftrightarrow f^{\prime}(x)$ actually involves a weak derivative. This is an important distinction in the case of the inverse stable density $h(x, t)$, because this density has a jump at the origin: $h(x, t)=0$ for $x<0$, but $h(0+, t)=t^{-\beta} / \Gamma(1-\beta)$, see Figure 2 .

\subsection{Mittag-Leffler eigenfunctions}

The Mittag-Leffler function

$$
E_{\beta}(z)=\sum_{j=0}^{\infty} \frac{z^{j}}{\Gamma(1+\beta j)}
$$

is a convergent power series for any complex $z$, which reduces to the exponential function when $\beta=1$. Mainardi and Gorenflo [18] prove that $E_{\beta}\left(\lambda t^{\beta}\right)$ is an eigenfunction of the Caputo fractional derivative (3.4) with eigenvalue $\lambda$, so that

$$
\partial_{t}^{\beta} E_{\beta}\left(\lambda t^{\beta}\right)=\lambda E_{\beta}\left(\lambda t^{\beta}\right) .
$$

Eigenfunctions are useful to solve ordinary differential equations. In Section 2, we used the eigenfunction relation $\partial_{t} e^{\lambda t}=\lambda e^{\lambda t}$ to solve the diffusion equation (2.2): Equation (2.3) states that $\hat{p}(k, t)$ is an eigenfunction of $\partial_{t}$ with eigenvalue $-D k^{2}$, hence we set $\hat{p}(k, t)=\exp \left(-t D k^{2}\right)$. Then we inverted the Fourier transform to arrive at (2.1). 
A similar argument has been applied to fractional diffusion problems [25]. First we consider the case of a traditional diffusion, to establish notation. Given a bounded domain $D$ in $\mathbb{R}^{d}$, under some technical conditions, the generator $L$ defined by (5.15) with Dirichlet boundary conditions $p(x, t)=0 \forall x \in \partial D$ has an orthonormal basis of eigenfunctions: $L \psi_{n}(x)=-\lambda_{n} \psi_{n}(x)$ on $L^{2}(D)$. Now the generalized diffusion equation

$$
\partial_{t} p(x, t)=L p(x, t) ; \quad p(x, 0)=f(x)
$$

can be solved by separation of variables. Assume $p(x, t)=v(x) w(t)$, so that (5.22) implies

$$
\frac{\partial_{t} w(t)}{w(t)}=\frac{L v(x)}{v(x)}=-\lambda .
$$

The eigenfunction solutions are $v_{n}(x)=\psi_{n}(x)$ and $w_{n}(t)=\exp \left(-\lambda_{n} t\right)$, leading to the general solution

$$
p(x, t)=\sum_{n=0}^{\infty} c_{n} \exp \left(-\lambda_{n} t\right) \psi_{n}(x),
$$

where $c_{n}=\int_{D} f(x) \psi_{n}(x) d x$. Solutions to the generalized time-fractional diffusion equation

$$
\partial_{t}^{\beta} q(x, t)=L q(x, t) ; \quad q(x, 0)=f(x)
$$

can be obtained by a very similar argument, substituting the Mittag-Leffler eigenfunctions for the exponential:

$$
q(x, t)=\sum_{n=0}^{\infty} c_{n} E_{\beta}\left(-\lambda_{n} t^{\beta}\right) \psi_{n}(x) .
$$

Bingham [6] showed that the inverse stable subordinator $E_{t}$ has a Mittag-Leffler distribution:

$$
\int_{0}^{\infty} e^{-s u} h(u, t) d u=E_{\beta}\left(-s t^{\beta}\right) .
$$

Then the solution to $(5.24)$ is

$$
\begin{aligned}
q(x, t) & =\sum_{n=0}^{\infty} c_{n} \psi_{n}(x) \int_{0}^{\infty} e^{-\lambda_{n} u} h(u, t) d u \\
& =\int_{0}^{\infty}\left(\sum_{n=0}^{\infty} c_{n} e^{-\lambda_{n} u} \psi_{n}(x)\right) h(u, t) d u
\end{aligned}
$$

which reduces to (2.7). This shows that $A\left(E_{t}\right)$ is the stochastic solution to (5.24). In the very special case $L=-\partial_{x}$ on an unbounded domain with initial condition $f(x)=\delta(x),(5.24)$ reduces to the governing equation (5.7) for the inverse stable density $h(x, t)$.

\section{Summary}

The inverse stable subordinator is an important and useful tool in the theory of time-fractional diffusion equations. The inverse stable process can be used as a time change, to develop stochastic solutions to time-fractional diffusion problems. The inverse stable density leads to explicit solution formulae for a broad range of time-fractional diffusion problems. The simplest of these governs the inverse stable density itself. Several different forms of that governing equation have appeared in the mathematics and physics literature. In order to reconcile these forms, it is necessary to distinguish the different kinds of ordinary and fractional derivatives (Riemann-Liouville and Caputo, weak and strong) that appear in those equations.

Acknowledgements. This research was partially supported by NSF grants DMS-1025486, DMS-0803360, and NIH grant R01-EB012079. 


\section{References}

[1] W. Arendt, C. J. K. Batty, M. Hieber,F. Neubrander. Vector-valued Laplace transforms and Cauchy problems, Monographs in Mathematics 96, Birkhäuser Verlag, Basel, 2001.

[2] B. Baeumer, M. M. Meerschaert. Stochastic solutions for fractional Cauchy problems. Fract. Calc. Appl. Anal. 4 (2001), $481-500$

[3] E. Barkai, R. Metzler, J. Klafter. From continuous time random walks to the fractional Fokker-Planck equation. Phys. Rev. E 61 (2000), 132.

[4] D.A Benson, M.M Meerschaert. A simple and efficient random walk solution of multi-rate mobile/immobile mass transport equations. Adv. Water Resour. 32 (2009), 532-539.

[5] B. Berkowitz, A. Cortis, M. Dentz, H. Scher. Modeling non-Fickian transport in geological formations as a continuous time random walk. Rev. Geophys. 44 (2006), 1-49.

[6] N. H. Bingham. Limit theorems for occupation times of Markov processes. Z. Wahrsch. verw. Geb. 17 (1971), 1-22.

[7] R. Durrett. Probability: Theory and Examples. Cambridge University Press, New York, 2010.

[8] A. Einstein. On the movement of small particles suspended in a stationary liquid demanded by the molecular kinetic theory of heat. Ann. Phys. 17 (1905), 549-560.

[9] W. Feller. An Introduction to Probability Theory and Its Applications. 2nd ed., Wiley, New York, 1971.

[10] M. G Hahn, K. Kobayashi, S. Umarov. Fokker-Planck-Kolmogorov equations associated with time-changed fractional Brownian motion. Proc. Amer. Math. Soc., 139 (2011), 691-705.

[11] N. Jacob. Pseudo differential operators and Markov processes. Vol. I. Imperial College Press, London, 2001.

[12] A. N. Kochubei. A Cauchy problem for evolution equations of fractional order. Diff. Eq. 25 (1989), 967-974.

[13] A. N. Kochubei. Fractional-order diffusion. Diff. Eq. 26 (1990), 485-492.

[14] V. N. Kolokoltsov. Generalized continuous-time random walks, subordination by hitting times, and fractional dynamics. Th. Prob. Appl. 53 (2009), 594.

[15] R.L. Magin. Fractional Calculus in Bioengineering. Begell House, 2006.

[16] M. Magdziarz, A. Weron. Competition between subdiffusion and Lévy flights: Stochastic and numerical approach. Phys. Rev. E 75 (2007), 056702.

[17] F. Mainardi. Fractals and fractional calculus in continuum mechanics. Springer Verlag, 1997.

[18] F. Mainardi, R. Gorenflo. On Mittag-Leffler-type functions in fractional evolution processes. J. Comput. Appl. Math. 118 (2000), 283-299.

[19] J. Masoliver, M. Montero, J. Perelló, G.H. Weiss, J. Perello. The continuous time random walk formalism in financial markets. J. Econ. Behav. Org. 61 (2006), 577-598.

[20] R. Metzler, J. Klafter. The random walk's guide to anomalous diffusion: a fractional dynamics approach. Phys. Rep. 339 (2000), 1-77.

[21] R. Metzler, J. Klafter. The restaurant at the end of the random walk: recent developments in the description of anomalous transport by fractional dynamics. J. Phys. A: Math. Gen. 37 (2004), R161-R208.

[22] M. M. Meerschaert, H.-P. Scheffler. Limit Distributions for Sums of Independent Random Vectors: Heavy Tails in Theory and Practice. Wiley, New York, 2001.

[23] M. M. Meerschaert, H.-P. Scheffler, P. Kern. Limit theorems for continuous-time random walks with infinite mean waiting times. J. Appl. Probab. 41 (2004), 455-466.

[24] M. M. Meerschaert, H.-P. Scheffler. Triangular array limits for continuous time random walks. Stoch. Proc. Appl. 118 (2008), 1606-1633.

[25] M. M. Meerschaert, E. Nane, P. Vellaisamy. Fractional Cauchy problems on bounded domains. Ann. Probab. 37 (2009), 979-1007.

[26] M. M. Meerschaert, A. Sikorskii. Stochastic Models for Fractional Calculus. De Gruyter, Berlin, 2012.

[27] R. Metzler, E. Barkai, J. Klafter. Deriving fractional Fokker-Planck equations from a generalised master equation. Europhys. Lett. 46 (1999), 431-436.

[28] E. Montroll, G. Weiss. Random walks on lattices. II. J. Math Phys. 6 (1965), 167-181.

[29] R. R. Nigmatullin. The realization of the generalized transfer in a medium with fractal geometry. Phys. Status Solidi B 133 (1986), 425-430.

[30] A. Pazy. Semigroups of Linear Operators and Applications to Partial Differential Equations, Applied Mathematical Sciences 44, Springer-Verlag, New York, 1983.

[31] A. Piryatinska, A. I. Saichev, W. Woyczynski. Models of anomalous diffusion: The subdiffusive case. Phys. A 349 (2005), 375-420.

[32] I. Podlubny. Fractional differential equations, Academic press, 1999.

[33] R Development Core Team. R: A Language and Environment for Statistical Computing, R Foundation for Statistical Computing, Vienna, Austria, 2010.

[34] J. Sabatier, O.P. Agrawal, J.A.T. Machado. Advances in Fractional Calculus: Theoretical Developments and Applications in Physics and Engineering. Springer, 2007.

[35] S. G. Samko, A. A. Kilbas, O. I. Marichev. Fractional Integrals and Derivatives: Theory and Applications. Gordon and Breach, London, 1993.

[36] E. Scalas. Five years of continuous-time random walks in econophysics. Complex Netw. Econ. Inter. 567 (2006), 3-16.

[37] A.I. Saichev, W.A. Woyzczynski. Distributions in the Physical and Engineering Sciences: Distributional and Fractal Calculus, Integral Transforms, and Wavelets. Birkhäuser, 1997. 
[38] A.I. Saichev, G.M. Zaslavsky. Fractional kinetic equations: solutions and applications. Chaos, 7 (1997), 753-764.

[39] H. Scher, M. Lax. Stochastic transport in a disordered solid. I. Theory. Phys. Rev. B 7 (1973), 4491-4502.

[40] W. R. Schneider, W. Wyss. Fractional diffusion and wave equations. J. Math. Phys. 30 (1989), $134-144$.

[41] I. N. Sneddon. Fourier Transforms. Dover, New York, 1995.

[42] I. Stakgold, M. J. Holst. Green's functions and boundary value problems. Wiley, New York, 1998.

[43] V. V. Uchaikin, V. M. Zolotarev. Chance and Stability. Stable Distributions and Their Applications. VSP, Utrecht, 1999.

[44] G. Zaslavsky. Fractional kinetic equation for Hamiltonian chaos. Phys. D 76 (1994), 110-122.

[45] Y. Zhang, D. Benson, M. M. Meerschaert, H. Scheffler. On using random walks to solve the space-fractional advectiondispersion equations. J. Stat. Phys. 123 (2006), 89-110.

[46] V. Zolotarev. One-dimensional Stable Distributions. Translations of Mathematical Monographs 65, American Mathematical Society, Providence, RI, 1986. 